\title{
Algorithm for designing a hydraulic scissor lifting platform
}

\author{
Cornel Ciupan ${ }^{1, *}$, Emilia Ciupan ${ }^{1}$, and Emanuela Pop $^{1}$ \\ ${ }^{1}$ Technical University of Cluj-Napoca, Memorandumului Street, No. 28, 400114 Cluj-Napoca, Cluj \\ County, Romania
}

\begin{abstract}
The paper describes the hydraulic scissor platforms and presents an algorithm for their design and optimization. Depending on the dimensions of the platform, the maximum height at which it rises and the maximum load is presented a program for calculating the number of scissors and the forces in the joints. The obtained data allow to choose the type of hydraulic table (with one, two or three scissors) and sizing the elements according to the beneficiary's requirements.
\end{abstract}

\section{Introduction}

Lifting work platforms are mechanical devices used for height vertical transport, also known as aerial work platforms (AWP). These platforms consist of a metal base and a metal platform, with which different loads can be lifted, controlling the platform ascent and descent [1].

Lifting platforms are used in many areas, for vertical lifting, for example outside buildings at various heights, warehouses, firefighters and emergency services, etc. because they are much more flexible than conventional lifts and elevators [2].

Depending on the application field of the platforms, there are several types of aerial platforms, elevating work platform (EWP), bucket truck or mobile elevating work platform (MEWP). The lifting platforms can be fixed or mobile, operated electro-hydraulically, electro-mechanical, pneumatic or hydraulic, using different lifting devices, such as chains, screw, rack, scissors etc. Stationary elevators are established in a defined place, frequently without the special foundation on a flat surface of a floor and fastened by means of anchor bolts or special pins. If the elevator is telescopic then for his installation a special basement is required. The main advantage of mobile elevators is their mobility - a possibility to use serially on various posts and in various technological zones of the enterprise [3].

Aerial devices are operated by hydraulic cylinders, mostly used for maintenance operations, indoors or outdoors, in areas where other equipment cannot be used. This kind of mechanisms can be lifted in different directions, up and over, due to the jointed sections [4].

The most common are the articulated elevated work platform EWP, being some mechanical systems of hydraulic levers and cylinders in the form of foldable support in a criss-cross pattern, called scissor mechanisms, which act to raise the platform only

* Corresponding author: cornel.ciupan@muri.utcluj.ro 
vertically [5]. The drive mechanism used to ascent or descent the platform can be hydraulic, pneumatic or mechanical. Scissors platforms can be also characterized by number of scissors, with one pair of scissors, two or more, used to rise different platforms at a certain high.

Scissors platform has many advantages, being versatile, economical, efficient, reliable lifting systems that require low maintenance. They can be easily configured to meet user requirements as well, are also systems that can be relocated easily.

In order to design a platform with scissors, the structure of the platform must be determined, the positioning of the scissors at the base of the platform and at the moving table, the hydraulic control parts and the driving of the hydraulic cylinder. The most important mechanism is the scissor lift mechanism, whose force directly influences the performance of the entire equipment and together with the positioning of the hydraulic cylinder determines lifting height of the platform [6].

\section{Design and optimization of hydraulic platforms}

\subsection{Description of scissors hydraulic platforms}

The double scissor lifting platform in figure 1 is composed of a base frame, which supports a movable upper platform, called the platform table, by means of two scissors. Each scissor consists of two "X" arms, mounted on both sides of the platform. The scissors at the base of the platform are provided with two joints, a fixed bearing (fixed support) and a mobile bearing (sliding support). At the top of the scissors is fixed by another scissors, in the case of platforms with several scissors. The last scissor is coupled to the mobile platform in a similar way to the base frame. The lifting of the platform is done with two hydraulic cylinders mounted between the stiffening bars between the scissors.

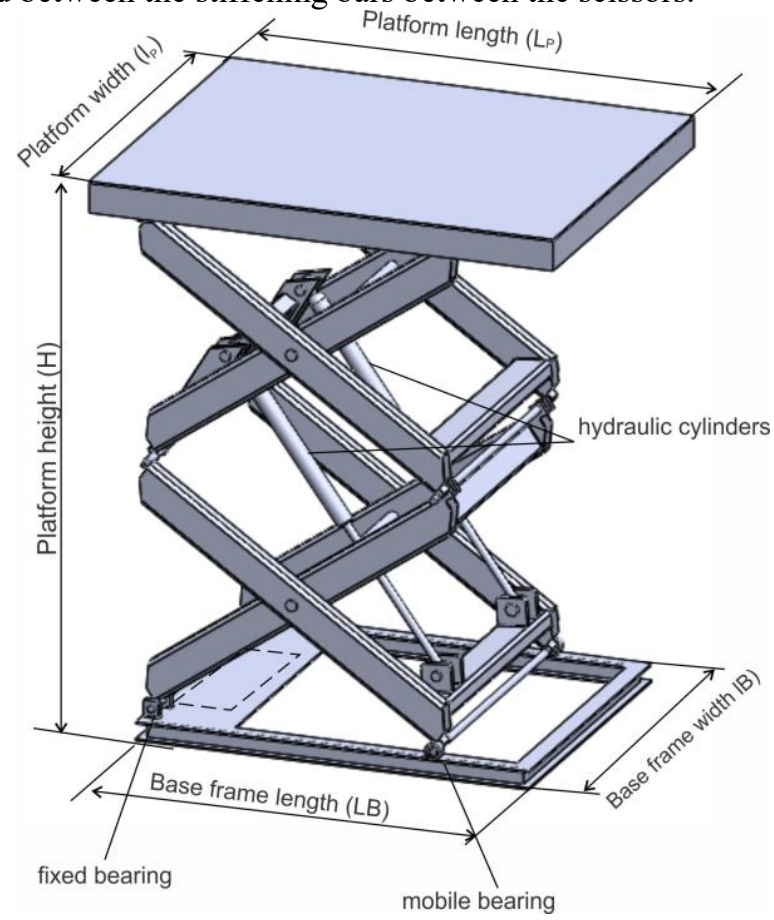

Fig. 1. Scissor hydraulic platform 


\subsection{Design and optimization algorithm for a hydraulic scissor platform}

The product design consists of several stages, according to figure 2, these being the market analysis, the elaboration of the design specifications, the conceptual design, the detailed and the technological design.

The market analysis phase aims to identify the needs of the users and the opportunities for developing new products. For the proposed product for development, the design specifications are elaborated, consisting of the functional, manufacturing, operational requirements and legal constraints.

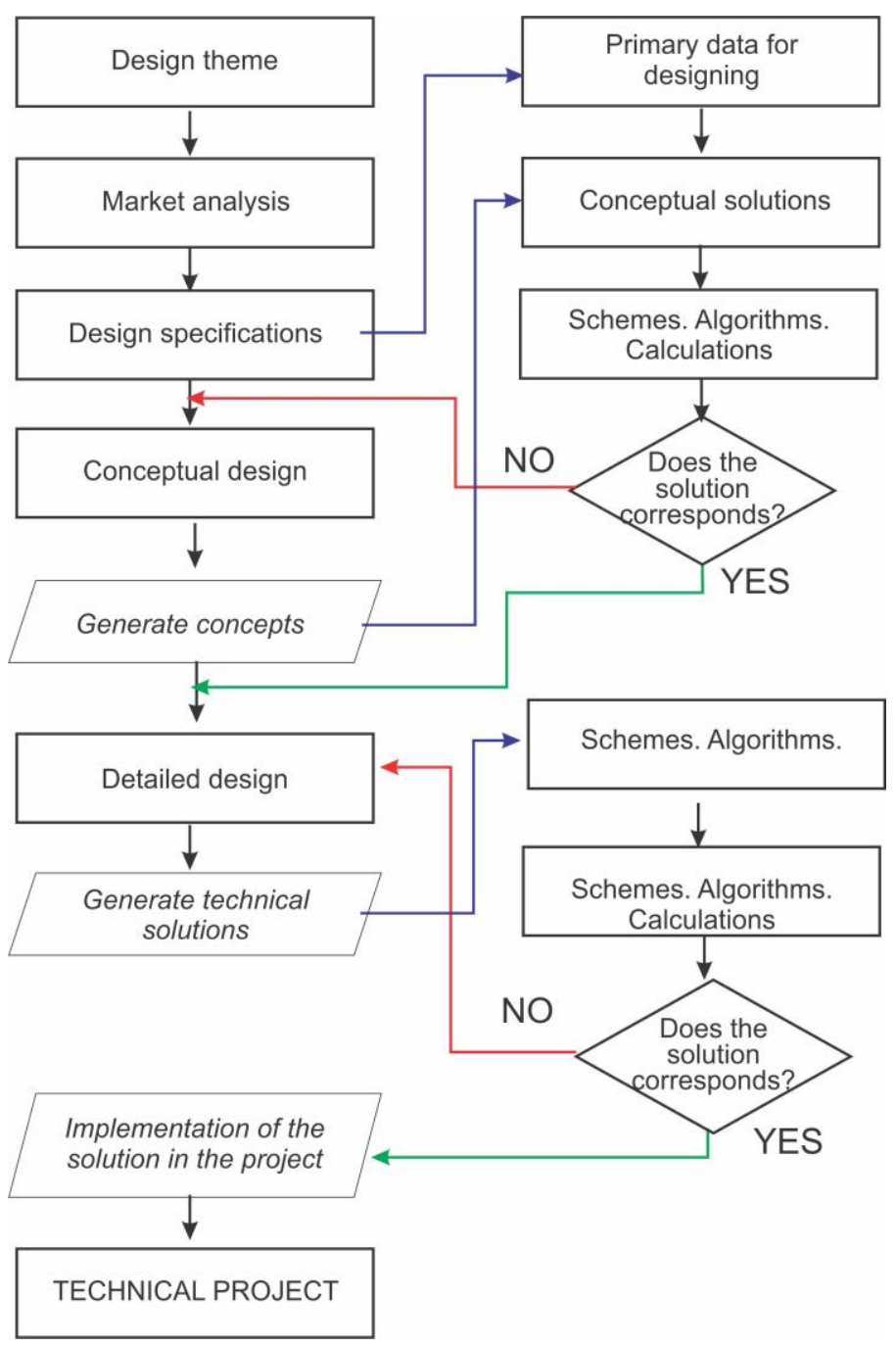

Fig. 2. Design and optimization algorithm

The conceptual design stage is one of the most important stages, which helps to choose the concept to be developed, offering the possibility of optimization in the situation when it does not correspond.

Once the final concept is chosen, we move on to the detailed design, which involves designing all the assemblies and subassemblies, making part drawings to be processed, 
establishing the transport, packaging, maintenance instructions, etc. After verification, if the product corresponds, the technical solutions are implemented in the project, resulting in the technical project and proceed to the design of the technological processes.

\section{Algorithm for determining the number of scissors}

In order to facilitate the design and optimization of lifting platforms, it is planned to perform a calculation program based on the input data: platform dimensions $\left(L_{P} \times l_{P}\right)$, lifting height $\left(\mathrm{H}_{\min }, \mathrm{H}_{\max }\right)$, maximum load $\left(\mathrm{P}_{\max }\right)$.

Depending on these data, it is first and foremost to determine the size of the scissors and their number. Once the structure of the platform has been established, one can move on to the calculation of the joint forces and the driving forces of the hydraulic motors. Finally the platform is designed according to the user's requirements, applications, dimensions, load, cost, but also the results of the calculations.

Based on figure 3, a program has been developed that allows the number of scissors to be determined, considering the following input data.

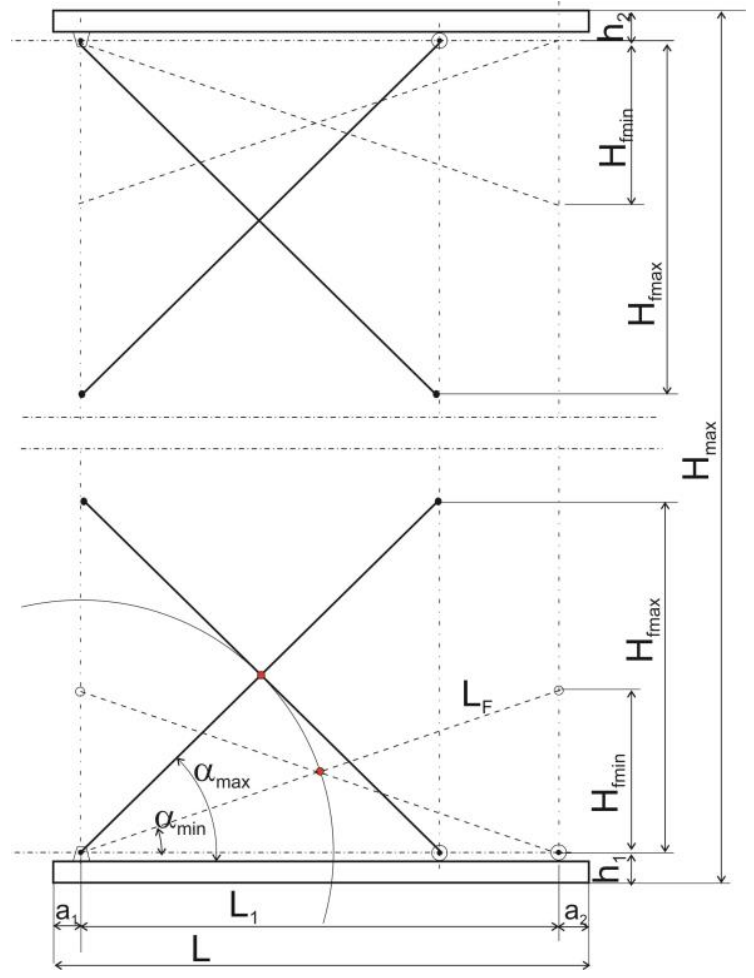

Fig. 3. Schematic diagram of a platform with $\mathrm{n}$ scissors

The input data for calculating the number of scissors are Platform length, (L) and maximum height, $\left(\mathrm{H}_{\max }\right)$.

The constructive data are, the minimum/ maximum (folded/open scissors) angle, $\alpha_{\min }$; $\alpha_{\max }\left[{ }^{\circ}\right]$ and cotes $a_{1}, a_{2}, h_{1}, h_{2}$ as in the figure 3 . The length of the scissors (between the axes of the extreme bearings) is $l_{l}=L_{F} / 2$ and can be calculated with the following relation: 


$$
l_{1}=\frac{L-\left(a_{1}+a_{2}\right)}{2 \cos \left(\alpha_{\min }\right)}
$$

The actual length of the scissors given by the relation (1) is rounded to the value $l$, depending on the semi-finished from which are manufactured or by the option of the designer.

The calculated number for the scissors is determined by the relation:

$$
n=\frac{H_{\max }-\left(h_{1}+h_{2}\right)}{2 \cdot l \cdot \sin \left(\alpha_{\max }\right)}
$$

The number of scissors $N$ is adopted, being the integer part of the value in the relation (2), rounded up, and then the minimum and maximum height is calculated according to $N$ and $\alpha_{\min }, \alpha_{\max }$.

$$
\begin{gathered}
H_{\text {max }}=h_{1}+h_{2}+2 N \cdot l \cdot \sin \left(\alpha_{\text {max }}\right) \\
H_{\text {min }}=h_{1}+h_{2}+2 N \cdot l \cdot \sin \left(\alpha_{\text {min }}\right)
\end{gathered}
$$

From the calculations the number of scissors required for lifting to the maximum height $\left(H_{\max }\right)$ results in a fractional number. In such situations, the designer can either choose for rounding up the number of scissors, increasing the lifting height or can change with the beneficiary's agreement the length of the table $(L)$, reducing the number of scissors and the cost of the platform.

\section{Program for determining the forces}

For the determination of the joint forces and the variation of the hydraulic motor force, depending on the angle $\alpha$, of the scissors, was used the scheme shown in figure 4.

For the calculation of the driving force of the hydraulic motor it is considered that the frictional forces are zero and the principle of energy conservation (mechanical work) is applied. Thus, it is considered that the mechanical work produced by the hydraulic motor is equal to the mechanical work required for lifting the load.

By solving the equilibrium relations of forces and moments, using figure 4 , a MATHCAD program was developed to solve the system of equations, and then a program for determining the forces in the joints and the resistant force to the hydraulic motor rod, a program that is presented below.

\subsection{Sizing calculations for a platform with double scissors}

Input data:

- Functional characteristics: $N ; H_{\max } ; H_{\min } ; P_{\max } ;$

- Constructive characteristics:

- location of hydraulic motor bearings relative to points $\mathrm{G}$ (base) and $\mathrm{F}$ (scissors) $\left(G_{x} ; G_{y} ; F_{x} ; F_{y}\right)$

- scissor length $l$

- scissors angle $\alpha$

- motor angle $\beta$ 
Calculation of the coordinates of points $\mathrm{G}$ and $\mathrm{F}$ :

$$
\begin{gathered}
X_{F}=F_{x} \cdot \cos \alpha-F_{y} \cdot \sin \alpha \\
Y_{F}=\left(2 l+F_{x}\right) \cdot \sin \alpha+F_{y} \cdot \cos \alpha \\
X_{G}=\left(2 l-G_{x}\right) \cos \alpha+G_{y} \cdot \sin \alpha \\
Y_{G}=G_{x} \cdot \sin \alpha+G_{y} \cdot \cos \alpha \\
\sin \beta=\frac{Y_{F}-Y_{G}}{\sqrt{\left(X_{G}-X_{F}\right)^{2}+\left(Y_{G}-Y_{F}\right)^{2}}} \\
\cos \beta=\frac{X_{G}-X_{F}}{\sqrt{\left(X_{G}-X_{F}\right)^{2}+\left(Y_{G}-Y_{F}\right)^{2}}}
\end{gathered}
$$

\subsection{Calculation of the forces in joints}

In order to calculate the forces in joints, in the case where equal forces are applied, $\mathrm{P}$, the following notations are defined:

- F- hydraulic motor force;

- VA, HA- the reactions in the fixed support A;

- VD, HD - the reactions in the sliding support D

- VE, HE- the reactions in the central joint $\mathrm{E}$,

$$
\begin{gathered}
F=\frac{2 \cdot P \cdot \cos \alpha \cdot l}{Y_{G} \cdot \cos \beta+X_{G} \cdot \sin \beta-2 \cdot l \cdot \sin \alpha \cdot \cos \beta} \\
V A=\frac{2 \cdot P \cdot X_{F} \cdot \sin \beta+P \cdot X_{G} \cdot \sin \beta+2 \cdot P \cdot Y_{F} \cdot \cos \beta+P \cdot Y_{G} \cdot \cos \beta}{X_{G} \cdot \sin \beta+Y_{G} \cdot \cos \beta-2 \cdot l \cdot \sin \alpha \cdot \cos \beta}+ \\
+\frac{-4 \cdot P \cdot l \cdot \cos \alpha \cdot \sin \beta-6 \cdot P \cdot l \cdot \sin \alpha \cdot \cos \beta}{X_{G} \cdot \sin \beta+Y_{G} \cdot \cos \beta-2 \cdot l \cdot \sin \alpha \cdot \cos \beta} \\
+\frac{2 \cdot P \cdot X_{G} \cdot \cos \alpha \cdot \sin \beta-2 \cdot P \cdot X_{F} \cdot \cos \alpha \cdot \sin \beta}{X_{G} \cdot \sin \alpha \cdot \sin \beta+Y_{G} \cdot \sin \alpha \cdot \cos \beta-2 \cdot l \cdot \sin 2 \alpha \cdot \cos \beta} \\
+\frac{-2 \cdot P \cdot Y_{F} \cdot \cos \alpha \cdot \cos \beta+2 \cdot P \cdot Y_{G} \cdot \cos \alpha \cdot \cos \beta}{X_{G} \cdot \sin \alpha \cdot \sin \beta+Y_{G} \cdot \sin \alpha \cdot \cos \beta-2 \cdot l \cdot \sin ^{2} \alpha \cdot \cos \beta} \\
+\frac{4 \cdot P \cdot l \cdot \cos \alpha \cdot \sin \alpha \cdot \cos \beta}{X_{G} \cdot \sin \alpha \cdot \sin \beta+Y_{G} \cdot \sin \alpha \cdot \cos \beta-2 \cdot l \cdot \sin ^{2} \alpha \cdot \cos \beta}
\end{gathered}
$$




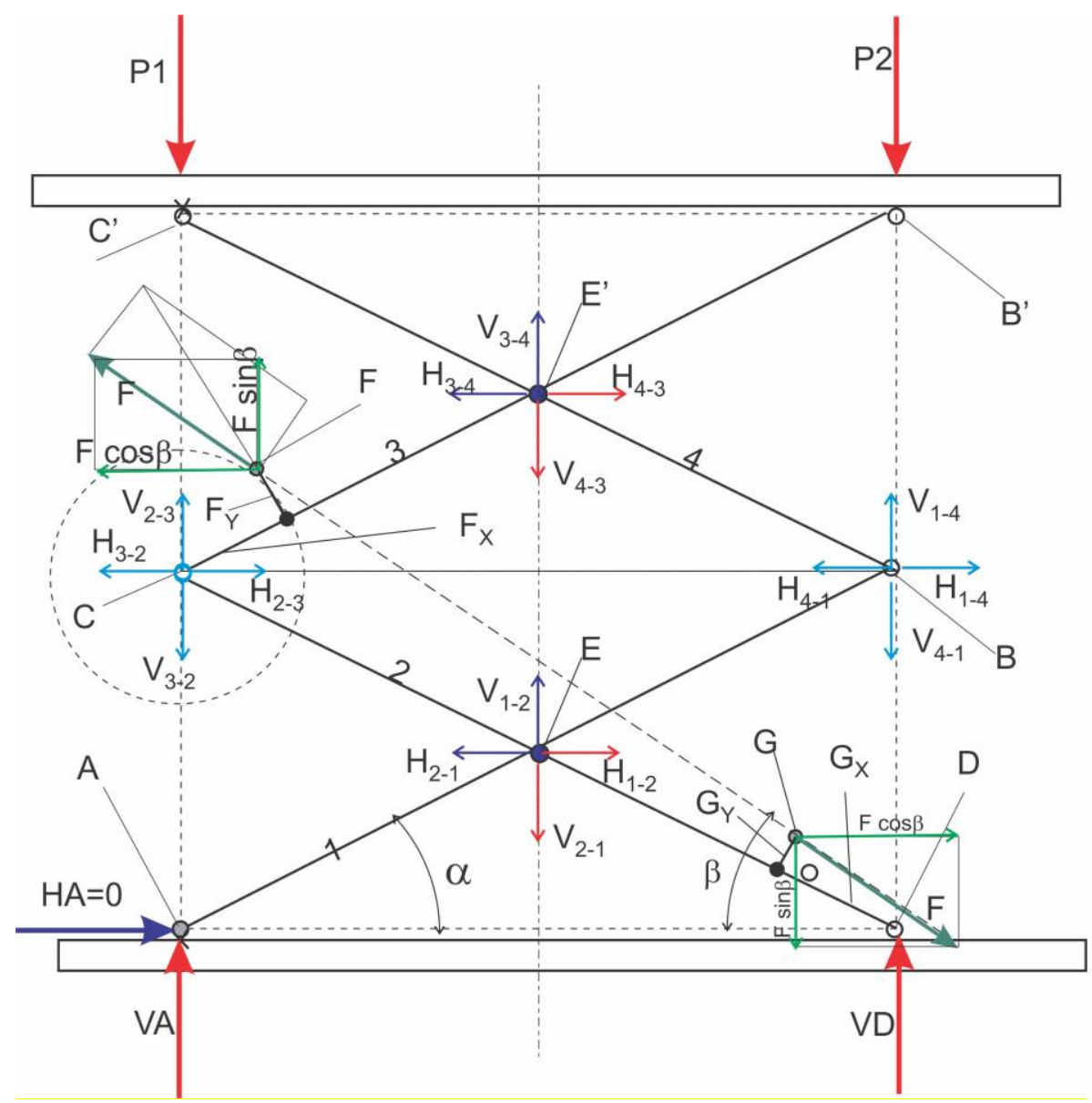

Fig. 4. Work forces scheme in case of hydraulic platform with double scissors

$$
\begin{gathered}
V D=\frac{P \cdot X_{G} \cdot \sin \beta-2 \cdot P \cdot X_{F} \cdot \sin \beta-2 \cdot P \cdot Y_{F} \cdot \cos \beta}{X_{G} \cdot \sin \beta+Y_{G} \cdot \cos \beta-2 \cdot l \cdot \sin \alpha \cdot \cos \beta} \\
+\frac{P \cdot Y_{G} \cdot \cos \beta+2 P \cdot l \cdot \sin \alpha \cdot \cos \beta}{X_{G} \cdot \sin \beta+Y_{G} \cdot \cos \beta-2 \cdot l \cdot \sin \alpha \cdot \cos \beta} \\
H D=-\frac{2 \cdot P \cdot X_{F} \cdot \cos \alpha \cdot \sin \beta-2 \cdot P \cdot X_{G} \cdot \cos \alpha \cdot \sin \beta}{X_{G} \cdot \sin \alpha \cdot \sin \beta+Y_{G} \cdot \sin \alpha \cdot \cos \beta-2 \cdot l \cdot \sin ^{2} \alpha \cdot \cos \beta}- \\
-\frac{2 \cdot P \cdot Y_{F} \cdot \cos \alpha \cdot \cos \beta-2 \cdot P \cdot Y_{G} \cdot \cos \alpha \cdot \cos \beta}{X_{G} \cdot \sin \alpha \cdot \sin \beta+Y_{G} \cdot \sin \alpha \cdot \cos \beta-2 \cdot l \cdot \sin ^{2} \alpha \cdot \cos \beta} \\
V E=-\frac{2 \cdot P \cdot X_{F} \cdot \sin \beta+2 \cdot P \cdot Y_{F} \cdot \cos \beta-4 \cdot P \cdot l \cdot \sin \alpha \cdot \cos \beta}{X_{G} \cdot \sin \beta+Y_{G} \cdot \cos \beta-2 \cdot l \cdot \sin \alpha \cdot \cos \beta}
\end{gathered}
$$




$$
\begin{aligned}
H E & =\frac{2 \cdot P \cdot X_{F} \cdot \cos \alpha \cdot \sin \beta-2 \cdot P \cdot X_{G} \cdot \cos \alpha \cdot \sin \beta}{X_{G} \cdot \sin \alpha \cdot \sin \beta+Y_{G} \cdot \sin \alpha \cdot \cos \beta-2 \cdot l \cdot \sin ^{2} \alpha \cdot \cos \beta}+ \\
& +\frac{2 \cdot P \cdot Y_{F} \cdot \cos \alpha \cdot \cos \beta-2 \cdot P \cdot Y_{G} \cdot \cos \alpha \cdot \cos \beta}{X_{G} \cdot \sin \alpha \cdot \sin \beta+Y_{G} \cdot \sin \alpha \cdot \cos \beta-2 \cdot l \cdot \sin ^{2} \alpha \cdot \cos \beta}
\end{aligned}
$$

\section{Case study}

Based on the relationships determined in previous chapters in the following part is presented a case study of designing a hydraulic platform with the following inputs:

- Platform length: $L=2500 \mathrm{~mm}$;

- Maximum Height: $H_{\max }=3800 \mathrm{~mm}$;

- Total force on the table $P_{\max }=4 \mathrm{P}=20000 \mathrm{~N}$;

- $a l=a 2=100 \mathrm{~mm} ; h l=h 2=80 \mathrm{~mm}$;

- The scissors angle: $\alpha_{\min }=10^{\circ} ; \alpha_{\max }=45^{\circ}$

- $G_{x}=100 \mathrm{~mm} ; G_{y}=50 \mathrm{~mm}$;

- $F_{x}=100 \mathrm{~mm} ; F_{y}=100 \mathrm{~mm}$;

The calculated length of the scissors is $l_{l}=1168 \mathrm{~mm}$ and the adopted length is $l=1170$ $\mathrm{mm}$ that correspond to the $n=2.2$ scissors.

After discussions with the beneficiary, the platform length is increased to $L^{\prime}=2800 \mathrm{~mm}$ and the scissor length is recalculated and adopted to $l=1300 \mathrm{~mm}$. The obtained number of scissors is $n^{\prime}=1.98 . N=2$ is adopted.

The following constructive values are obtained from the program:

$H$ min $=1063 \mathrm{~mm}$;

Hmax $=3837 \mathrm{~mm}$

The forces resulting in the joints are shown in Figures 5-7.

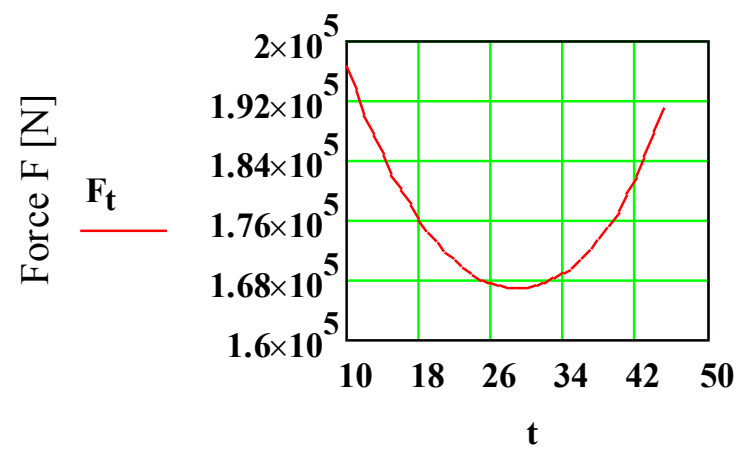

Scissor angle $\left[{ }^{\circ}\right]$

Fig. 5. The variation of the force according to scissor angle $\alpha$ (in the program $t=\alpha$ ) 


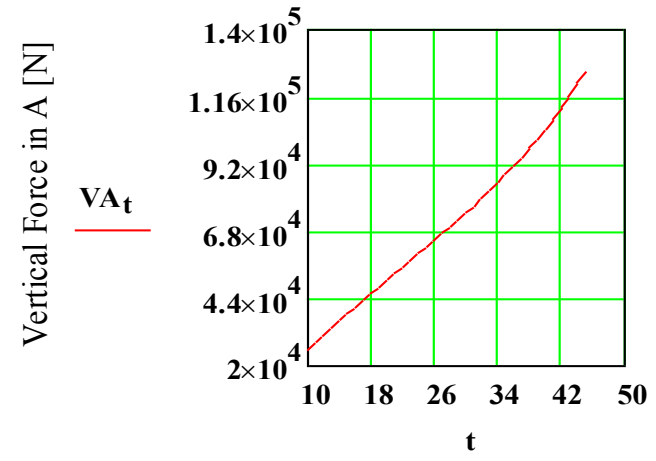

Scissor angle $\left[^{\circ}\right]$

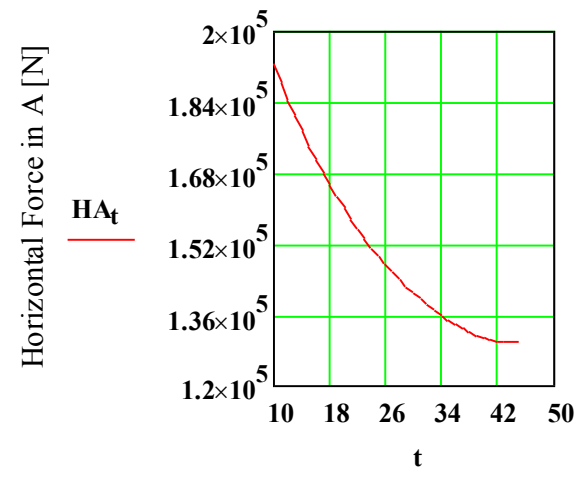

Scissor angle $\left[{ }^{\circ}\right]$

Fig. 6. The variation of the force in fixed support A (in the program $t=\alpha$ )

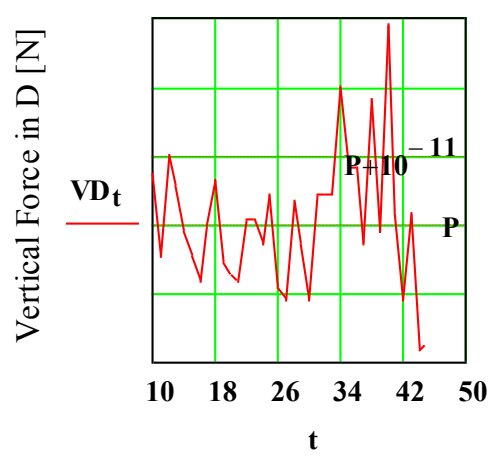

Scissor angle $\left[{ }^{\circ}\right]$

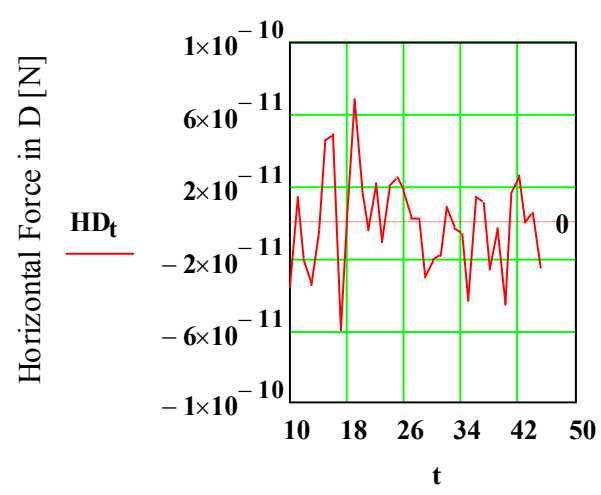

Scissor angle $\left[{ }^{\circ}\right]$

Fig. 7. The variation of the force in fixed support $D$ (in the program $t=\alpha$ )

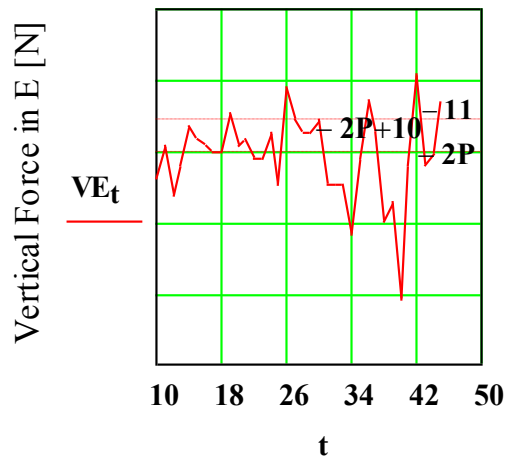

Scissor angle $\left[{ }^{\circ}\right]$

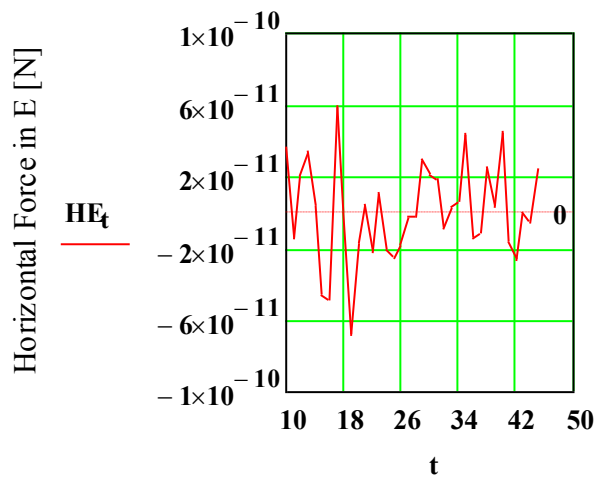

Scissor angle $\left[{ }^{\circ}\right]$

Fig. 8. The variation of the force in central joint $E$ (in the program $t=\alpha$ ) 


\section{Conclusions}

The algorithm offers the possibility to optimize the design of scissor lifting tables, according to the functional requirements imposed by the beneficiary and the specific application at their place of use.

Based on the algorithm, a MATHCAD program was developed which allows in the first stage the determination of the number of scissors and their length, and then the calculation of the forces in the joints, necessary for dimensioning. The force at the hydraulic motor shaft and the forces at the scissor joints are dependent on the scissor angle. The maximum values of the forces are useful for dimensioning the constructive elements of the tables and for choosing the hydraulic actuating cylinders.

In the case study, the stages of designing a table have been completed, for a given application, validating the calculation program. In the first stage the number of scissors and their length were determined. By the fact that the beneficiary accepted the increase of the table length from $2500 \mathrm{~mm}$ to $2800 \mathrm{~mm}$, it was possible to reduce the number of scissors to 2 , otherwise being forced to design a table with three scissors to obtain the maximum lifting height. By reducing the scissors number it is also reduced the height of the table in folded state $\left(\mathrm{H}_{\min }\right)$, which allowed it to fit into the functional requirements without requiring the placement of the base below the floor level. These elements are relevant to reducing costs.

In the second stage the forces in the joints and from the motor shaft were determined. It was found that the maximum force to be developed by the hydraulic motor $\left(\mathrm{F}_{\max }=\right.$ $196800 \mathrm{~N}$ ) is obtained at the minimum scissor angle, and this decreases by approximately $20 \%$ at the half cylinder travel. The force of the motor is strongly influenced by the positioning of points $\mathrm{F}$ and $\mathrm{G}$, but their position is also restricted by hydraulic cylinder travel.

\section{References}

1. R.K.. Barsel, Fluid Mechanics, 2nd Edition, John Wiley \& Sons, (1998)

2. R.K. Gupta, Machine Design, 4th Edition, Eurasia Publishing House, Ltd., (2006)

3. T. Hongyu, Z. Ziyi, Design and Simulation Based on Pro/E for a Hydraulic Lift Platform in Scissors Type, International Workshop on Automobile, Power and Energy Engineering, Procedia Engineering 16 ( 2011 ) 772 - 781

4. https://en.wikipedia.org/wiki/Aerial_work_platform

5. D. Rani, N. Agarwal, V. Tirth, Design and Fabrication of Hydraulic Scissor Lift, MIT International Journal of Mechanical Engineering, Vol. 5, No. 2, pp. 81-87, (2015)

6. G. Olenin, Design of hydraulic scissors lifting platform, Saimaa University of Applied Sciences Faculty of Technology, Lappeenranta, Thesis, (2016) 